\title{
Scheduling Markovian PERT networks to maximize the net present value: New results
}

\author{
Ben Hermans ${ }^{\mathrm{a}}$, Roel Leus ${ }^{\mathrm{a}, *}$ \\ ${ }^{a}$ Research Center for Operations Research 83 Business Statistics (ORSTAT), Faculty of Economics and Business, \\ $K U$ Leuven, Belgium
}

\begin{abstract}
We study project scheduling so as to maximize the expected net present value when task durations are exponentially distributed. Based on the structural properties of an optimal solution we show that, even if preemption is allowed, it is not necessary to do so. Next to its managerial importance, this result also allows for a new algorithm which improves on the current state of the art with several orders of magnitude, both in CPU time and in memory usage.
\end{abstract}

Keywords: project scheduling, net present value, exponentially distributed activity durations, Markov decision process, monotone optimal policy

\section{Introduction}

Consider a project in which a set of tasks $N=$ $\{1, \ldots, n\}$ needs to be performed in order to reach a given goal such as, for example, the development of a new product. The strict partial order $A \subset N \times N$ defines precedence constraints, with $(i, j) \in A$ indicating that task $j$ can only start if $i$ is finished. We suppose task $n$ reflects the project's completion and $(i, n) \in A$ for all $i \in N \backslash\{n\}$. Each task $i \in N$ has a random duration $\tilde{d}_{i}$ with support $\mathbb{R}_{\geq 0}$ and, initially, we require activities to be processed without interruption. Finally, each task has a cash flow which is discounted to take into account the time value of money.

In this article, we study the problem of deciding when to start each task so as to maximize the project's expected net present value (eNPV), i.e., the expected sum of discounted cash flows. Throughout, we assume independent and exponentially distributed activity durations $\left(\tilde{d}_{i}\right)_{i \in N}$ with rate parameter $\left(\lambda_{i}\right)_{i \in N} \in \mathbb{R}_{>0}^{n}$. This problem was also studied by $[1,2,9]$; we briefly review these articles below. For an excellent and more detailed literature review, also for the case where task durations are not exponentially distributed, see [11].

\footnotetext{
${ }^{*}$ Corresponding author. Faculty of Economics and Business, KU Leuven, Naamsestraat 69, 3000 Leuven, Belgium.

Email address: roel.leus@kuleuven. be (Roel Leus)
}

All models in $[1,2,9]$ are based on the seminal work of Kulkarni and Adlakha [6], who use a continuous-time Markov chain to evaluate the moments and distribution of the project's earliest completion time. Buss and Rosenblatt [1] adapt this Markov chain to evaluate the eNPV when each task is initiated as soon as possible. Next, they determine the optimal delay for up to two activities beyond their earliest possible starting time. While the delays in [1] are fixed before the project's start, Sobel et al. [9] consider the more general case of making scheduling decisions adaptively during the project's execution, solving the problem using a stochastic dynamic program (SDP). To mitigate the SDP's excessive memory usage, Creemers et al. [2] partition the state space such that not all states have to be stored in memory at the same time. This significantly improves performance and their algorithm is considered to be the current state of the art [11].

We address the same problem as [2], which we define in Section 2 and for which we give a new SDPformulation in Section 3. The major difference is that we act as if activities can be interrupted, but show that, even if preemption is allowed, it is not necessary to do so (Section 4). Consequently, the preemptive case solves the non-preemptive case as well. Next to its managerial importance, this result also allows for a new algorithm that improves on 
the method of [2] with several orders of magnitude, both in CPU time and in memory usage (Section 5). As in [9], our proofs exploit the structural properties of the SDP's value function. Additionally, however, we also examine the structural properties of an optimal solution and employ the concept of supermodularity; a property underlying numerous monotonicity proofs $[7,10]$.

\section{Problem definition}

We assume that executing task $i \in N$ leads to a cash flow $c_{i} \in \mathbb{R}$ per time unit, such that $c_{i} \tilde{d}_{i}$ equals the activity's total cash flow. Applying a continuous discount rate $r \in \mathbb{R}_{>0}$ to task $i$ starting at time $\tau$ then yields a net present value

$$
\int_{\tau}^{\tau+\tilde{d}_{i}} c_{i} e^{-r t} \mathrm{~d} t
$$

We make the, often realistic, assumption that $c_{i} \leq 0$ for $i \in N \backslash\{n\}$ and $c_{n}>0$; in a new product development project, for example, the company typically only obtains a revenue after launching the product [1].

A solution is a policy that specifies which task to start at which decision moment, possibly depending on events predating this decision moment. Denote the set of all feasible policies by $\Pi$, then any policy $\pi \in \Pi$ maps realizations $\boldsymbol{d}:=\left(d_{i}\right)_{i \in N}$ of the random durations $\tilde{\boldsymbol{d}}:=\left(\tilde{d}_{i}\right)_{i \in N}$ into starting times $\left(s_{i}(\boldsymbol{d})\right.$ $\pi))_{i \in N}$. To be feasible, policy $\pi$ has to respect the precedence constraints: $s_{i}(\boldsymbol{d} \mid \pi)+d_{i} \leq s_{j}(\boldsymbol{d} \mid \pi)$ for any $(i, j) \in A$ and any realization $\boldsymbol{d}$ of $\tilde{\boldsymbol{d}}$. Moreover, the non-anticipativity constraint states that, when making a decision, the project manager cannot use information that becomes available after the decision moment. Formally, this means that, for any two realizations $\boldsymbol{d}$ and $\boldsymbol{d}^{\prime}$ of $\tilde{\boldsymbol{d}}$, we have $s_{i}(\boldsymbol{d} \mid \pi)=s_{i}\left(\boldsymbol{d}^{\prime} \mid \pi\right)$ as long as $d_{j}=d_{j}^{\prime}$ for any $j \in N$ with $s_{j}(\boldsymbol{d} \mid \pi)+d_{j} \leq s_{i}(\boldsymbol{d} \mid \pi)$. We obtain the following problem:

$$
\max _{\pi \in \Pi} \mathbb{E}\left[\sum_{i \in N} \int_{s_{i}(\tilde{\boldsymbol{d}} \mid \pi)}^{s_{i}(\tilde{\boldsymbol{d}} \mid \pi)+\tilde{d}_{i}} c_{i} e^{-r t} \mathrm{~d} t\right] .
$$

Note that [2] assume fixed cash flows $c_{i}^{F}$ occurring at task $i$ 's initiation. Such cash flows can be incorporated into our setting by choosing $c_{i}$ such that $c_{i}^{F}=\mathbb{E}\left[\int_{0}^{\tilde{d}_{i}} c_{i} e^{-r t} \mathrm{~d} t\right]$. Indeed, as can be derived from the expression above, this leads to the

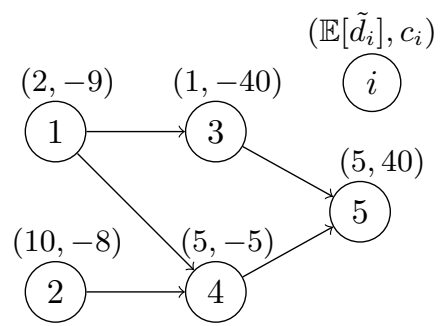

Figure 1: Example project.

same decisions and eNPV, see [9, 11]. We model the cash flows as rates because this feels more natural for the preemptive case.

Example. Figure 1 visualizes an example project consisting of five activities $N=\{1, \ldots, 5\}$ represented on the nodes and precedence constraints as implied by the arrows. The arc $1 \rightarrow 3$, for instance, indicates that task 3 can only start after activity 1's completion. The expected task durations $\mathbb{E}\left[\tilde{d}_{i}\right]=1 / \lambda_{i}$ and cash flow rates $c_{i}$ are shown above the nodes. Together with a continuous discount rate $r=0.01$, this information fully specifies a problem instance.

One possible solution is the early-start policy $\pi^{e}$, which initiates each task as soon as possible. Using the procedure of [1], we find that the eNPV for $\pi^{e}$ equals 13.49 , while optimally delaying task 3 by 3.57 time units leads to an eNPV of 14.07. Using the SDP of [9], finally, we find that the maximum eNPV equals 16.59 ; the optimal policy $\pi^{\star}$ starts each activity different from task 3 as soon as possible and, for task 3, waits until tasks 1, 2 and 4 are past.

\section{Stochastic dynamic program}

In this section, we formulate a new stochastic dynamic program which assumes that we can interrupt a task at any time during its execution. In Section 4, we show that an optimal policy for this preemptive case solves the non-preemptive case as well and, thus, our SDP effectively gives a formulation for the problem of Section 2.

We define the project's state at any given time by the set $P \subseteq N$ of activities that are already finished (past). The project's state space $\mathcal{P}$ collects all states $P \subseteq N$ such that $i \in P$ implies $j \in P$ for all $(j, i) \in A$. The terminal state $P=N$ represents a completed project. The major difference 


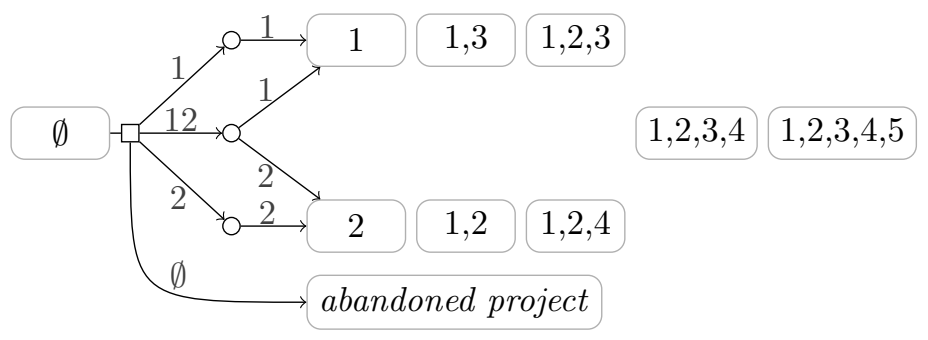

Figure 2: Illustration of states and transitions.

compared to the SDPs of $[2,9]$ is that our state definition does not include the set of active tasks, i.e., tasks that are in process. Since preemption is allowed, we can choose this set at any moment anew and the additional information is no longer needed. As will become clear, this significantly reduces the SDP's number of states and, thus, its memory requirements.

For any state $P \in \mathcal{P}$, define the set of eligible tasks $E(P)$ as the set of tasks which, in principle, could be active. Formally, $E(P)=\{i \in N \backslash P \mid j \in$ $P$ for all $(j, i) \in A\}$. A possible action then is to work on a set of tasks $\varphi \subseteq E(P)$. Choosing $\varphi=\emptyset$ when $P \neq N$ corresponds to project abandonment: we never execute the remaining tasks $N \backslash P$ and the project transitions to an additional terminal state that we simply call 'abandoned project'. Section 4.3 discusses the changes if abandonment is not allowed.

It follows from [3] that there is no loss in optimality when choosing actions at state transitions only. Either it is desirable to work on task $i \in E(P)$ in state $P \in \mathcal{P}$ and we do so during the entire visit of state $P$, or it is not desirable and we do not work on task $i$ at all in state $P$. Intuitively speaking, the exponential distribution's memoryless property implies that as long as no transition occurs, one obtains no additional information that could alter the desirability of working on task $i$. This considerably simplifies the problem since we can limit decision moments to a discrete set of state transitions, while, in general, decisions could be made at any time, i.e., continuously, during the project's execution.

Suppose we choose action $\varphi \subseteq E(P)$ in state $P \in \mathcal{P}$, then the next state transition occurs randomly depending on which of the subsequently active tasks $i \in \varphi$ completes first. By the memoryless property of the exponential distribution, the remaining duration of each active task $i \in \varphi$ is exponentially distributed with parameter $\lambda_{i}$. The time until the next transition is the minimum of these durations and, thus, is exponentially distributed with parameter $\Lambda_{\varphi}:=\sum_{i \in \varphi} \lambda_{i}$. Moreover, the probability that $i \in \varphi$ completes first equals $\lambda_{i} / \Lambda_{\varphi}$ and is independent from the time until the next transition. Finally, it is easily verified that the expected discount rate until the next state transition equals $\Lambda_{\varphi} /\left(r+\Lambda_{\varphi}\right)$ and that the expected cash flow incurred during the state's visit equals $\sum_{i \in \varphi} c_{i} /\left(r+\Lambda_{\varphi}\right)$.

Example. Figure 2 displays the possible sets of past activities $P \in \mathcal{P}$ for the example project of Figure 1. The node "1,3", for instance, represents state $P=\{1,3\}$. In addition, there is a schematic representation of the possible transitions in the initial state. The square represents a decision node, indicating that the possible actions are the subsets of $E(\emptyset)=\{1,2\}$. After choosing action $\varphi=\{1,2\}$, the chance node (circle) indicates that the next transition occurs randomly depending on whether task 1 or 2 completes first. To save space, we display the possible actions and transitions for the initial state only.

Define the value function $V(\cdot)$ as the mapping that assigns to each state $P \in \mathcal{P}$ the maximum eNPV that is attainable by the remaining activities when the tasks in $P$ are past. It then follows from [8] that $V(\cdot)$ satisfies the Optimality Equation

$$
V(P)=\max _{\varphi \subseteq E(P)}\left\{\frac{\sum_{i \in \varphi} \lambda_{i} V\left(P_{i}\right)+c_{i}}{r+\sum_{i \in \varphi} \lambda_{i}}\right\}
$$

and that choosing actions which attain the maximum constitute an optimal policy. Here, and in the sequel, we denote $P_{i}:=P \cup\{i\}, P_{i j}:=P \cup\{i, j\}$ and so forth. Since every task completion increases the cardinality of $P$ with one unit, a backward recursion with boundary condition $V(N)=0$ identifies $V(P)$. Our goal is to compute $V(\emptyset)$ and to identify a policy which attains this maximum. 
Let $m:=\max _{P \in \mathcal{P}}|E(P)|$ denote the maximum number of tasks that can be active in parallel, then it is not difficult to show that $|\mathcal{P}|$ is bounded above by $\sum_{i=0}^{m}\left(\begin{array}{c}n \\ i\end{array}\right)$; see [4]. Indeed, $P$ is completely determined by $E(P)$ and $\left(\begin{array}{c}n \\ i\end{array}\right)$ bounds the number of states having $|E(P)|=i$. In fact, by the binomial theorem, $(1+n)^{m}=\sum_{i=0}^{m}\left(\begin{array}{c}m \\ i\end{array}\right) n^{i} \geq \sum_{i=0}^{m} n^{i} \geq$ $\sum_{i=0}^{m}\left(\begin{array}{c}n \\ i\end{array}\right)$ and the number of states is polynomial in $n$ for a fixed value of $m$. Since the completion of every subset $\varphi \subseteq E(P)$ leads to a feasible state $P \cup \varphi$, we also have $|\mathcal{P}| \geq 2^{m}$, implying that the number of states is exponential in $m$. If preemption is not allowed, we need to include the set of active tasks in each state [9]. Since all $Y \subseteq E(P)$, i.e., $2^{|E(P)|}$ subsets for each $P \in \mathcal{P}$, are possible sets of active tasks, the number of states for the nonpreemptive case is bounded above by $\sum_{i=0}^{m}\left(\begin{array}{c}n \\ i\end{array}\right) 2^{i}$ and bounded below by $3^{m}$. This suggests that the preemptive case is computationally more tractable than the non-preemptive one.

\section{Structural properties}

In the first part of this section, we characterize an optimal preemptive policy with a structure that facilitates the recursive computation of a state's value. Next, we use this structure to show how this optimal preemptive solution solves the nonpreemptive case as well. Finally, we discuss the changes if project abandonment is not allowed. We will frequently use the following simple equivalence between (in)equalities:

Lemma 1. For any $u, v \in \mathbb{R}$ and $w, \lambda \in \mathbb{R}_{>0}$ holds that

$$
v \geq \frac{u}{w} \Longleftrightarrow \frac{u+\lambda v}{w+\lambda} \geq \frac{u}{w} \Longleftrightarrow v \geq \frac{u+\lambda v}{w+\lambda},
$$

and likewise for "=" or " $\leq$ " instead of " $\geq$ ".

\subsection{Structure of an optimal preemptive policy}

Remember that $c_{i}$ is the cash flow per time unit for working on task $i \in N . V\left(P_{i}\right)$, in turn, equals the value when $i$ completes, while $\lambda_{i}$ is the rate at which the task completes. Consequently, we can interpret $\lambda_{i} V\left(P_{i}\right)$ as the value per time unit generated by the possible completion of task $i$. Now define

$$
\psi_{i}(P):=\frac{1}{\lambda_{i}}\left(\lambda_{i} V\left(P_{i}\right)+c_{i}\right)=V\left(P_{i}\right)+\frac{c_{i}}{\lambda_{i}},
$$

then, since $1 / \lambda_{i}$ equals task $i$ 's expected duration, $\psi_{i}(P)$ reflects the total expected value of working on task $i$. Finally, denote

$$
\varphi^{\star}(P):=\left\{i \in E(P) \mid \psi_{i}(P) \geq V(P)\right\},
$$

then the next result states that we should work on task $i$ whenever the total expected value of working on $i$ is not less than the current value $V(P)$.

Proposition 1. Let $P \in \mathcal{P}$, then $\varphi^{\star}(P)$ is an optimal action in state $P$.

Proof. Given a state $P \in \mathcal{P}$, consider an eligible task $i \in E(P)$ and action $\varphi \subseteq E(P)$ such that $i \notin \varphi$. From the first equivalence in Lemma 1 ,

$$
\psi_{i}(P) \geq \frac{\sum_{j \in \varphi} \lambda_{j} \psi_{j}(P)}{r+\sum_{j \in \varphi} \lambda_{j}}
$$

if and only if

$$
\frac{\sum_{j \in \varphi} \lambda_{j} \psi_{j}(P)+\lambda_{i} \psi_{i}(P)}{r+\sum_{j \in \varphi} \lambda_{j}+\lambda_{i}} \geq \frac{\sum_{j \in \varphi} \lambda_{j} \psi_{j}(P)}{r+\sum_{j \in \varphi} \lambda_{j}} .
$$

By Optimality Equation $(1), V(P)$ equals the maximum of Inequality (2)'s right-hand side over all actions $\varphi \subseteq E(P)$ and, thus, working on task $i$ never decreases value if $\psi_{i}(P) \geq V(P)$. Similarly, from Lemma 1's second equivalence, an optimal action never includes task $i$ if $\psi_{i}(P)<V(P)$.

While the result of Proposition 1 is intuitively appealing, it does not help from a computational point of view. Indeed, when searching for the optimal action in state $P$ we obviously do not know the value $V(P)$ yet. The next result expresses $\varphi^{\star}(P)$ in terms of the value function evaluated at sets of past activities with cardinality $|P|+1$. As such, it is useful for the backward recursion. For any $P \in \mathcal{P}$ and $i \in E(P)$, define

$$
\tau_{i}(P):=\frac{\sum_{j \in E(P): \psi_{j}(P)>\psi_{i}(P)} \lambda_{j} V\left(P_{j}\right)+c_{j}}{r+\sum_{j \in E(P): \psi_{j}(P)>\psi_{i}(P)} \lambda_{j}},
$$

then $\tau_{i}(P)$ can be interpreted as the value of choosing action $\varphi=\left\{j \in E(P) \mid \psi_{j}(P)>\psi_{i}(P)\right\}$ in Optimality Equation (1). The intuition behind Theorem 1 is as follows. Since $\psi_{i}(P)$ reflects the value of working on $i$, we prefer to work on tasks having higher $\psi_{i}(P)$. The result then states that we want to 'add' task $i$ to the set of active tasks if working on $i$ does not decrease value compared to the situation where we only work on tasks $j$ with $\psi_{j}(P)>\psi_{i}(P)$. 
Theorem 1. Let $P \in \mathcal{P}$, then $\varphi^{\star}(P)=$ $\left\{i \in E(P) \mid \psi_{i}(P) \geq \tau_{i}(P)\right\}$.

Proof. It suffices to show that, for each $P \in \mathcal{P}$ and $i \in E(P)$, it holds that $\psi_{i}(P) \geq V(P)$ if and only if $\psi_{i}(P) \geq \tau_{i}(P)$. The result that $\psi_{i}(P) \geq V(P)$ implies $\psi_{i}(P) \geq \tau_{i}(P)$ immediately follows from the fact that $V(P) \geq \tau_{i}(P)$. Indeed, $\{j \in E(P)$ | $\left.\psi_{j}(P)>\psi_{i}(P)\right\}$ is feasible for Optimality Equation (1) and leads to value $\tau_{i}(P)$.

For the other direction, i.e., that $\psi_{i}(P) \geq \tau_{i}(P)$ implies $\psi_{i}(P) \geq V(P)$, define the set $L:=\{j \in$ $\left.E(P) \mid \psi_{j}(P) \geq \tau_{j}(P)\right\}$ and a permutation $\rho$ on $L$ such that $\psi_{\rho(l)} \geq \psi_{\rho(l+1)}$ for all $l=1, \ldots,|L|-1$. Induction on $l$ will show that $\psi_{j}(P) \geq V(P)$ for all $j \in L$.

Suppose that $\psi_{\rho(1)}(P)<V(P)$, then, by definition of $\rho, \psi_{j}(P)<V(P)$ for all $j \in L$. Moreover, by definition of $L$ and the first part, $\psi_{j}(P)<V(P)$ for all $j \in E(P) \backslash L$ as well. Proposition 1 then implies that $\varphi^{\star}(P)=\emptyset$ and $V(P)=0$. Together with $0=\tau_{\rho(1)} \leq \psi_{\rho(1)}(P)$ this gives the contradiction: $0 \leq \psi_{\rho(1)}<V(P)=0$. Consequently, $\psi_{\rho(1)}(P) \geq \bar{V}(P)$

Now take arbitrary $k \in\{2, \ldots,|L|\}$ and assume $\psi_{\rho(l)}(P) \geq V(P)$ for $l<k$. The induction step consists of showing that $\psi_{\rho(k)}(P) \geq V(P)$ as well. If not, then similar reasoning as above yields $\psi_{\rho(l)}<V(P)$ for all $l=k+1, \ldots,|L|$ and $\psi_{j}<V(P)$ for $j \in E(P) \backslash L$. Since $\psi_{\rho(k)}<\psi_{\rho(k-1)}$, the induction hypothesis and Proposition 1 imply $V(P)=\tau_{\rho(k)}(P)$ and, together with $\tau_{\rho(k)} \leq$ $\psi_{\rho(k)}(P)$, this gives a contradiction. Consequently, $\psi_{\rho(k)}(P) \geq V(P)$.

\subsection{Optimality of a non-preemptive policy}

Not exercising preemption can be seen as a monotonicity in the actions: if we decide to work on task $i \in E(P)$ in state $P \in \mathcal{P}$, we want to work on it in any successor state $P_{j}$ with $j \in E(P) \backslash\{i\}$ as well. Such monotonicity would imply that an optimal preemptive solution solves the non-preemptive case as well. This is useful for at least two reasons. First, from a computational point of view, the preemptive case is easier to deal with (see Section 3 ). In addition, from a managerial point of view, it is interesting to know under which circumstances it is not useful to consider preemption.

The remainder of this section shows that a monotone preemptive policy is indeed optimal. First, Lemma 2 gives the intuitively obvious result that, since $c_{i} \leq 0$ for $i \in N \backslash\{n\}$, the completion of an intermediary task does not decrease the value function. Lemma 3, in turn, delivers the key step by showing that the change in value thanks to a task's completion does not decrease if another task finishes first; it proves that $V(\cdot)$ is supermodular. The proof for Lemma 2 is relatively straightforward and the one for Lemma 3 rather lengthy. They are therefore not included in this article but can be found in the working paper [5].

Lemma 2. Let $P \in \mathcal{P}$ with $n \notin E(P)$, then for any $i \in E(P): V\left(P_{i}\right) \geq V(P)$.

Lemma 3. Let $P \in \mathcal{P}$ with $|E(P)| \geq 2$, then for any $i, j \in E(P)$ with $i \neq j: V\left(P_{i j}\right)-V\left(P_{j}\right) \geq$ $V\left(P_{i}\right)-V(P)$.

Proposition 2. Let $P \in \mathcal{P}$ with $|E(P)| \geq 2$, then for any $i, j \in E(P)$ with $i \neq j: i \in \varphi^{\star}(P)$ implies $i \in \varphi^{\star}\left(P_{j}\right)$.

Proof. By definition of $\varphi^{\star}(P)$ and $\psi_{i}(P)$, it holds that $i \in \varphi^{\star}(P)$ if and only if $V\left(P_{i}\right)-V(P) \geq$ $-c_{i} / \lambda_{i}$. This implies $i \in \varphi^{\star}\left(P_{j}\right)$ as well since, by Lemma $3, V\left(P_{i j}\right)-V\left(P_{j}\right) \geq V\left(P_{i}\right)-V(P) \geq$ $-c_{i} / \lambda_{i}$.

Theorem 2. $\varphi^{\star}(\cdot)$ fully determines an optimal non-preemptive policy.

Proof. From Proposition 2, $\varphi^{\star}(\cdot)$ never interrupts a task which was active in a previous state. Consequently, the resulting policy is non-preemptive and, thus, is optimal for the non-preemptive case as well.

\subsection{Project abandonment}

From Lemma 2, $V(P)>0$ for all $P \in \mathcal{P}$ if $V(\emptyset)>0$. Thus, either we never abandon the project, or we do not initiate it. This result was also proposed by Sobel et al. [9, Proposition 2]; Lemma 2 gives an alternative proof.

One could think of situations, however, where we want to know the maximum eNPV, even if it is negative. This means that choosing $\varphi=\emptyset$ in Optimality Equation (1) is forbidden. In order to deal with this situation, let $P \in \mathcal{P}$ and take arbitrary

$$
i^{\star}(P) \in \underset{i \in E(P)}{\arg \max }\left\{\frac{\lambda_{i} V\left(P_{i}\right)+c_{i}}{r+\lambda_{i}}\right\} .
$$

Next, consider the action

$$
\bar{\varphi}^{\star}(P):= \begin{cases}i^{\star}(P) & \text { if } \psi_{i}(P) \leq 0 \text { for all } i \in E(P) ; \\ \varphi^{\star}(P) & \text { otherwise. }\end{cases}
$$


It then follows that $\bar{\varphi}^{\star}(\cdot)$ describes a policy where we perform the tasks sequentially as long as the value function is negative and switch to $\varphi^{\star}(\cdot)$ as soon as the value function becomes positive. The next proposition shows that $\bar{\varphi}^{\star}(\cdot)$ is optimal if it is not allowed to abandon the project.

Proposition 3. Let $P \in \mathcal{P}$, then $\bar{\varphi}^{\star}(P)$ is an optimal action in state $P$ if choosing $\varphi=\emptyset$ is forbidden. Moreover, $\bar{\varphi}^{\star}(\cdot)$ is non-preemptive.

Proof. If $\psi_{i}(P) \leq 0$ for all $i \in E(P)$, then

$$
\psi_{i}(P)=\frac{\lambda_{i} V\left(P_{i}\right)+c_{i}}{\lambda_{i}} \leq \frac{\lambda_{i} V\left(P_{i}\right)+c_{i}}{r+\lambda_{i}}
$$

and the optimality of initiating task $i^{\star}(P)$ follows from the definition of $i^{\star}(P)$ in combination with the first equivalence of Lemma 1 .

On the other hand, if $\psi_{i}(P)>0$ for some $i \in$ $E(P)$, then $V(P)>0$ and applying Lemma 2 yields $V\left(P^{\prime}\right)>0$ for all successor states $P^{\prime}$ of $P$ as well. Consequently, no abandonment will occur in the remainder of the project and Proposition 1 proves the optimality of $\varphi^{\star}(P)$.

Finally, to see that $\bar{\varphi}^{\star}(\cdot)$ is non-preemptive, first note that $\bar{\varphi}^{\star}(\cdot)$ only initiates a single activity if $\psi_{i}(P) \leq 0$ for all $i \in E(P)$; this task will be finished in the next state and no preemption occurs. Next, if $\psi_{i}(P)>0$ for some $i \in E(P)$, non-preemption is guaranteed by Proposition 2 .

\section{Algorithm and performance}

Algorithm 1 presents our new procedure to identify a project's maximum eNPV for both the non-preemptive and preemptive case. The set $\mathcal{P}_{k}$ collects all states $P \in \mathcal{P}$ having exactly $k \in\{0,1, \ldots, n\}$ completed tasks and, thus, $\left(\mathcal{P}_{0}, \mathcal{P}_{1}, \cdots, \mathcal{P}_{n}\right)$ forms a partition of $\mathcal{P}$. Note that Sobel et al. [9] also use this approach to partition the state space into different stages. As suggested by Kulkarni and Adlakha [6], this allows to significantly reduce the memory requirements since, after having evaluated the value function for all states in $\mathcal{P}_{k-1}$, we no longer need the information in $\mathcal{P}_{k}$.

In the remainder, we compare the performance of Algorithm 1 with the procedure of Creemers et al. [2] based on a number of test instances grouped by their value of $n$ and order strength $(O S)$. The latter measures the project network's density and equals the ratio of the number of pairs in $A$, i.e., $|A|$, and the maximum number of such

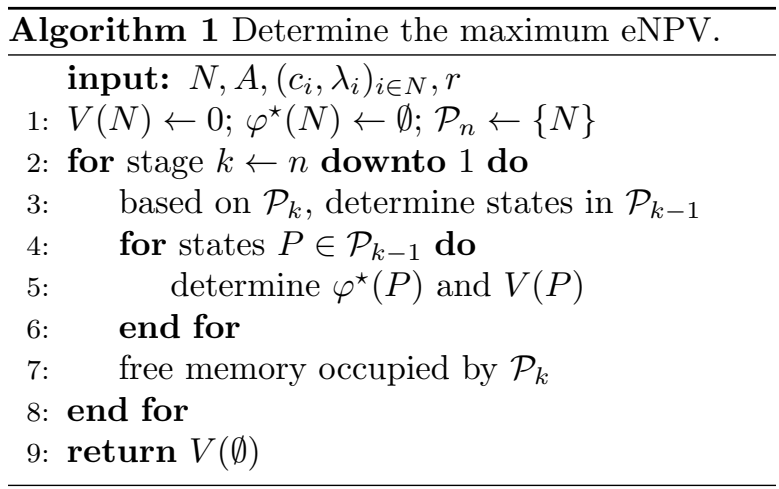

pairs, i.e., $n(n-1) / 2$. We reuse the dataset of [2], who generated 30 instances for each combination of $n \in\{10,20, \ldots, 120\}$ and $O S \in\{0.8,0.6,0.4\}$. We follow [2] by not allowing for project abandonment.

All experiments were performed with an Intel Core i7-4790 processor with $3.60 \mathrm{GHz}$ CPU speed and we used an upper bound of $1 \mathrm{~GB}$ of RAM. The upper part of Table 1 shows the average CPU time in seconds and the number of instances solved without violating the memory constraint. The lower part, in turn, displays the average total number of states as well as the average maximal fraction of these states kept in memory at the same time. This fraction measures how effectively the state space's partitioning mitigates memory requirements. All averages are taken over solved instances only.

Given the discussion in Section 3, it is not surprising that $O S$ is a major determinant of performance for both methods since a sparser network allows for more tasks executable in parallel. Our method clearly improves on the procedure of [2] with several orders of magnitude, both in memory usage and CPU time. To explain the reduced memory requirements, remember from Section 3 that the bounds on the number of states for our SDP are considerably lower than the ones for the method of [2]. It is interesting to note that the fraction of states in memory for our method is not only lower, but is also less sensitive to the order strength. This results from the fact that, contrary to the method of [2], Algorithm 1's partitioning of the state space does not depend on the number of tasks executable in parallel. Finally, to explain the reduction in CPU time, note that, in addition to the decrease in computational effort thanks to the reduced number of states, Theorem 1 enables to determine $\varphi^{\star}(P)$ without enumerating all subsets of $E(P)$. Since memory usage rather than CPU time still constitutes the 
Table 1: Average CPU time in seconds and number (\#) of solved instances out of 30 (upper part). Average total number (\#) of states and average maximal fraction (\%) of states in memory (lower part).

\begin{tabular}{|c|c|c|c|c|c|c|c|c|c|c|c|c|}
\hline \multirow[b]{3}{*}{$n$} & \multicolumn{6}{|c|}{ Creemers et al. [2] } & \multicolumn{6}{|c|}{ Algorithm 1} \\
\hline & \multicolumn{2}{|l|}{$O S=0.80$} & \multicolumn{2}{|c|}{$O S=0.60$} & \multicolumn{2}{|l|}{$O S=0.40$} & \multicolumn{2}{|c|}{$O S=0.80$} & \multicolumn{2}{|l|}{$O S=0.60$} & \multicolumn{2}{|c|}{$O S=0.40$} \\
\hline & $\mathrm{CPU}$ & $\#$ & $\mathrm{CPU}$ & $\#$ & $\mathrm{CPU}$ & $\#$ & $\mathrm{CPU}$ & $\#$ & $\mathrm{CPU}$ & $\#$ & $\mathrm{CPU}$ & \# \\
\hline 10 & 0.00 & 30 & 0.00 & 30 & 0.00 & 30 & 0.00 & 30 & 0.00 & 30 & 0.00 & 30 \\
\hline 20 & 0.00 & 30 & 0.01 & 30 & 0.27 & 30 & 0.00 & 30 & 0.00 & 30 & 0.00 & 30 \\
\hline 30 & 0.00 & 30 & 0.19 & 30 & 15.71 & 30 & 0.00 & 30 & 0.00 & 30 & 0.02 & 30 \\
\hline 40 & 0.02 & 30 & 3.84 & 30 & $1,213.43$ & 29 & 0.00 & 30 & 0.01 & 30 & 0.31 & 30 \\
\hline 50 & 0.08 & 30 & 50.70 & 30 & $11,287.77$ & 2 & 0.00 & 30 & 0.06 & 30 & 3.95 & 30 \\
\hline 60 & 0.42 & 30 & $1,288.93$ & 30 & & 0 & 0.01 & 30 & 0.29 & 30 & 58.96 & 30 \\
\hline 70 & 1.63 & 30 & $5,230.08$ & 17 & & 0 & 0.01 & 30 & 1.52 & 30 & 420.62 & 26 \\
\hline 80 & 6.06 & 30 & $25,628.35$ & 2 & & 0 & 0.03 & 30 & 5.72 & 30 & & 0 \\
\hline 90 & 27.60 & 30 & & 0 & & 0 & 0.06 & 30 & 38.58 & 30 & & 0 \\
\hline 100 & 98.09 & 30 & & 0 & & 0 & 0.12 & 30 & 151.44 & 30 & & 0 \\
\hline 110 & 590.56 & 30 & & 0 & & 0 & 0.30 & 30 & 501.76 & 27 & & 0 \\
\hline 120 & $2,495.16$ & 26 & & 0 & & 0 & 1.18 & 30 & $1,473.39$ & 10 & & 0 \\
\hline$n$ & \# states & $\%$ & \# states & $\%$ & \# states & $\%$ & \# states & $\%$ & \# states & $\%$ & \# states & $\%$ \\
\hline 10 & 71 & 40 & 206 & 52 & 695 & 57 & 22 & 29 & 41 & 32 & 84 & 33 \\
\hline 20 & 484 & 35 & 4,006 & 46 & 55,016 & 58 & 88 & 21 & 330 & 21 & 1,620 & 24 \\
\hline 30 & 1,995 & 29 & 49,388 & 42 & $1,560,364$ & 50 & 254 & 14 & 1,898 & 16 & 17,096 & 17 \\
\hline 40 & 7,860 & 29 & 534,014 & 41 & $47,072,515$ & 48 & 662 & 12 & 9,480 & 13 & 193,848 & 14 \\
\hline 50 & 26,667 & 31 & $4,346,215$ & 41 & $348,684,057$ & 23 & 1,544 & 11 & 40,379 & 12 & $1,659,705$ & 12 \\
\hline 60 & 92,003 & 32 & $42,278,506$ & 41 & & & 3,564 & 10 & 175,288 & 11 & $13,790,968$ & 11 \\
\hline 70 & 286,831 & 32 & $165,870,016$ & 36 & & & 7,754 & 10 & 727,948 & 10 & $77,917,666$ & 9 \\
\hline 80 & 829,741 & 28 & $603,402,153$ & 20 & & & 16,364 & 8 & $2,259,476$ & 8 & & \\
\hline 90 & $2,596,419$ & 31 & & & & & 34,057 & 8 & $9,845,427$ & 9 & & \\
\hline 100 & $6,868,100$ & 34 & & & & & 66,670 & 8 & $31,268,023$ & 8 & & \\
\hline 110 & $24,235,588$ & 34 & & & & & 146,910 & 8 & $90,918,894$ & 7 & & \\
\hline 120 & $112,181,874$ & 25 & & & & & 515,785 & 7 & $239,953,385$ & 5 & & \\
\hline
\end{tabular}

algorithm's bottleneck, further research should focus on identifying additional properties which could mitigate the algorithm's memory usage.

\section{Concluding remarks}

Note that Algorithm 1 does not output an optimal policy as this requires specifying an optimal action for every state and, thus, a prohibitively high memory usage. A pragmatic approach would be to work with a rolling horizon; the decision maker could, for instance, store the optimal action for every state in the next 20 stages and re-execute the algorithm after 10 stages. The appropriate horizon length then strikes a balance between memory usage and computation time.

While the models of Sobel et al. [9] and Creemers et al. [2] can incorporate general cash flows, we assume all intermediate cash flows to be non-positive. The reason is that Lemma 2 does not hold for tasks with a positive cash flow and, thus, if abandonment is not allowed, an initially positive value function may become negative as the project evolves. Preemption might then be optimal in order to 'postpone' the remaining project's negative eNPV. If project abandonment is allowed, however, then the eNPV is always non-negative and it is optimal to start each task with a positive cash flow as early as possible. In this latter case, the obtained results do carry over and we can use Algorithm 1.

Finally, in practice, the assumption of exponentially distributed tasks durations may not always be realistic. While Sobel et al. [9] address this issue by using independent phase-type distributions, this approach will generally not work in our algorithm because preemption might be optimal for non-exponentially distributed task durations. A possible direction for further research is to examine for which distributions non-preemption is still optimal. Here, the class of distributions with a nonincreasing 'mean residual lifetime' form a potential candidate.

\section{Acknowledgments}

The research was funded by a $\mathrm{PhD}$ Fellowship of the Research Foundation - Flanders. 


\section{References}

[1] A. H. Buss and M. J. Rosenblatt. Activity delay in stochastic project networks. Operations Research, 45 (1):126-139, 1997.

[2] S. Creemers, R. Leus, and M. Lambrecht. Scheduling Markovian PERT networks to maximize the net present value. Operations Research Letters, 38(1):51-56, 2010.

[3] E. A. Feinberg. Continuous time discounted jump Markov decision processes: a discrete-event approach. Mathematics of Operations Research, 29(3):492-524, 2004.

[4] E. Gutin, D. Kuhn, and W. Wiesemann. Interdiction games on Markovian PERT networks. Management Science, 61(5):999-1017, 2014.

[5] B. Hermans and R. Leus. Scheduling Markovian PERT networks to maximize the net present value: new results. KU Leuven - Faculty of Economics and Business. FEB Research Report KBI_1709. https://dx.doi.org/ 10.2139/ssrn.3052286, 2017.

[6] V. G. Kulkarni and V. G. Adlakha. Markov and Markov-regenerative PERT networks. Operations Research, 34(5):769-781, 1986 .

[7] M. L. Puterman. Markov Decision Processes: Discrete Stochastic Dynamic Programming. John Wiley \& Sons, 1994.

[8] R. F. Serfozo. Technical note - an equivalence between continuous and discrete time Markov decision processes. Operations Research, 27(3):616-620, 1979.

[9] M. J. Sobel, J. G. Szmerekovsky, and V. Tilson Scheduling projects with stochastic activity duration to maximize expected net present value. European Journal of Operational Research, 198(3):697 - 705, 2009.

[10] D. M. Topkis. Minimizing a submodular function on a lattice. Operations Research, 26(2):305-321, 1978.

[11] W. Wiesemann and D. Kuhn. The stochastic timeconstrained net present value problem. In Handbook on Project Management and Scheduling, volume 2, pages 753-780. Springer, 2015. 